\title{
Stochastic Treatment of Conformational Transitions of Polymer Chains in the Sub-Rouse Regime
}

\author{
Ivet Bahar* and Burak Erman
}

Polymer Research Center and School of Engineering, Bogazici University, Bebek 80815, Istanbul, Turkey

\section{Lucien Monnerie}

Laboratoire de Physicochimie Structurale et Macromoléculaire, associé au CNRS, ESPCI, 10 rue Vauquelin, 75231 Paris, Cedex 05, France

Received July 27, 1990; Revised Manuscript Received December 28, 1990

\begin{abstract}
Orientational autocorrelations and cross-correlations are considered for vectors rigidly affixed to bonds subject to configurational transitions in a long polymer chain. Transitions of bonds from one rotational isomeric state to the other are assumed to be dependent on the state of the neighboring bonds. Bond transition rates are obtained from Kramers' expression in the high-friction limit. The friction coefficient affecting a transition is assumed to depend on the location of the bond along the moving sequence in the chain. The joint probability of having a sequence of bonds in one configuration at time zero and in another at time $\tau$ and the orientational correlation functions are obtained by an efficient matrix multiplication scheme analogous to the matrix generator formalism of the rotational isomeric theory of chain statistics. A sequence of bonds whose length is prescribed by the time window of the experimental technique used is defined as an independent kinetic unit. The stochastic behavior of the latter is assumed to be uncorrelated with the remaining parts of the chain. Calculations are performed for different lengths of independent units, ranging from a few skeletal bonds to segments of the size of a Rouse subchain. Frequency distribution of relaxational modes obtained in this manner agree closely with previous calculations of Fixman by Langevin dynamics. Thus, unlike the Rouse dynamics predictions, the fastest modes of the investigated sub-Rouse regime scale linearly with inverse chain length and the distribution of relaxational frequencies for a given sequence exhibits a pronounced plateau.
\end{abstract}

\section{Introduction}

The description of the motions of a long chain in terms of $N$ stochastically independent Gaussian segments constitutes the basis of the bead-and-spring model of chain dynamics. According to this model, the chains are conveniently represented by a succession of $(N+1)$ beads joined together by Hookean springs subject to entropically driven elastic forces. In the original version introduced by Rouse, ${ }^{1}$ the hydrodynamic interaction is disregarded; the time evolution of the position of the beads obeys the linearized Langevin equation, a stochastic differential equation accounting for (i) the harmonic interaction potential between consecutive beads along the chain and (ii) the random motion of the beads characteristic of Brownian particles. This model has been widely used for analyzing low-frequency motions of polymers. In particular, it has proved to satisfactorily describe the viscoelastic behavior of polymer melts and semidilute solutions where the hydrodynamic interactions are effectively screened out. In the presence of hydrodynamic interactions, such as in the case of dilute solutions, it is rather replaced by its improved version, the Zimm model. ${ }^{2}$ The mathematical foundations of the Rouse-Zimm model and their generalizations may be found in the works of Bixon, ${ }^{3}$ Zwanzig, and Doi and Edwards. ${ }^{5}$

Although the concept of "stochastically uncorrelated Gaussian subchains" of the bead-and-spring model has lead to considerable progress in our understanding of chain dynamics, it suffers from the usual limitations of any mathematical model of wide generality. In fact, in the case of processes where the chemical structure and the detailed conformational characteristics of the materials become important, the Rouse-Zimm model is no longer applicable. In the extreme situation where the behavior is exclusively dominated by such short-range effects, recourse to models faithful to real chain characteristics at the repeat unit level becomes obligatory. In this smallwavelength limit, the few bonds involved in the local dynamics constitute a sequence much shorter than the stochastically independent Rouse subchain. All postulates of the Rouse-Zimm model fail in this limit. For a realistic estimation of the rates and mechanisms of such smallwavelength motions associated with rotameric transitions of backbone bonds, a plausible approach is the adoption of the fundamental postulates and approximations of the rotational isomeric state (RIS) model. The dynamic rotational isomeric state (DRIS) approach, ${ }^{6,7}$ developed along these lines, is applicable to high-frequency motions involving a few skeletal bonds undergoing rotameric transition, as illustrated by its recent application ${ }^{8}$ to NMR relaxation measurements in dilute polymeric solutions.

The time scale of the molecular motions involved in NMR relaxation processes lies approximately in the range $10^{-11}-10^{-10} \mathrm{~s}$, while Rouselike motions typically encountered in viscoelastic measurements, dielectric relaxation experiments, etc. are slower by about 2 or 3 orders of magnitude. In addition to their distinct time scale, highfrequency and low-frequency motions are distinguished by their qualitative dependence on the size of the moving segment. In fact, in Rouse dynamics, in which a diffusioncontrolled regime prevails, the relaxation times scale as $M^{2}$, where $M$ is the polymer molecular weight, whereas high-frequency or small-wavelength motions are independent of the size of the molecule inasmuch as they are highly localized along the chain: they are mainly governed 
by short-range conformational energetics. It can even be shown that with decreasing size of the moving unit, the diminishing frictional effect may be more than counterbalanced by the opposite "internal viscosity" effect, first pointed out by Kuhn, ${ }^{9}$ which is manifested by a progressive reduction in mobility accompanying the smaller number of degrees of freedom (or fewer pathways to relaxation). ${ }^{10,11}$

On the other hand, a large number of experiments such as neutron scattering, fluorescence anisotropy decay, and cyclization dynamics explore the intermediate frequency range, involving the cooperative motion of a group of skeletal bonds, forming a segment not large enough to be considered as a Gaussian subchain exhibiting Rouselike dynamics but, at the same time, comprising a definitely sufficient number of mobile bonds to experience substantial frictional resistance constraining the motion. It is that intermediate frequency domain that will be the object of the present study. A weaker dependence on the size of the kinetic unit, compared to Rouselike dynamics, is expected to take place in that intermediate regime. Indeed, the Langevin dynamics simulations of constrained chain stochastics carried out some time ago by Fixman ${ }^{12,13}$ clearly demonstrate the differences between the Rouse modes and those high-frequency motions that belong to what will be referred to as the sub-Rouse regime in the following. In fact, through introduction of hindered rotational barriers, the terminal mode relaxation rates exhibit a linear dependence on chain length, as shown by Fixman.

We note that the intermediate regime dynamics has been investigated by Allegra and collaborators as well, ${ }^{10,14-17}$ in their interpretation of quasi-elastic neutron scattering experiments. Although the classical linear Langevin equation with the Fourier representation of dynamical modes is used in their study, in parallel with the RouseZimm model, they depart in their definition of intramolecular elasticity. The latter is accounted for through adoption of realistic quadratic approximations for the potential between all pairs of atoms instead of the ends of the Gaussian subchains of the Rouse-Zimm model. Accordingly, molecular structural and conformational characteristics predicted by the RIS scheme are utilized for both (i) the evaluation of the generalized characteristic ratio determining the elastic contribution to motion and (ii) the estimation of the effect of internal viscosity associated with energy barriers to bond isomeric rotations. Inasmuch as the specific chain properties are considered, the theory is particularly suitable to correct or complement the higher frequency modes of Rouselike dynamics and has proved to satisfactorily explain the intermediatewavelength motions involved in neutron scattering experiments. 16,17

In the following, an alternate approach is proposed for the exploration of the intermediate regime dynamics. The study is essentially motivated by the simulations of Fix$\operatorname{man}^{12,13}$ where the intermediate regime dynamics are investigated. Specifically, we address the validity of extending the DRIS calculations into the intermediate range of frequencies. The DRIS model, originally developed for highly localized motions and hence emphasizing the role of short-range intramolecular conformational potential, assigns a mean frictional resistance to motion regardless of the size of the connected moving units accompanying a given bond rotation. However, it is evident that motions involving large-scale reorientations or cooperative rearrangements along the chain occur at slower rates due to higher experienced frictional resistance. In fact, the latter is operative in the damping of the propagation of reorientation, as one proceeds away from

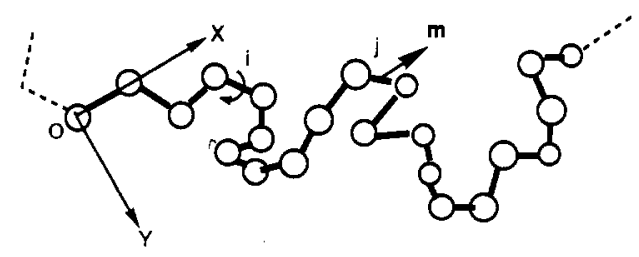

Figure 1. Schematic representation of a given kinetic unit of 21 bonds. The bonds within the unit are subject to coupled conformational dynamics. The motions of the chain segments beyond the ends of the unit are assumed to be stochastically uncorrelated to those within the unit. The rotameric transition takes place over the $i$ th bond, the vector $\mathbf{m}$ is affixed to the $j$ th bond. $O X Y Z$ represents the molecule fixed coordinate system with the $X Y$ plane coinciding with the first two bonds of the kinetic unit. For clarity the $Z$-axis, which completes a right-handed system, is not shown.

a rotating bond, along the chain. In other words, upon the rotameric jump of a given bond, the newly induced orientation is not affinely transmitted/reflected upon the neighboring bonds but instead undergoes some gradual attenuation manifested by the reducea mobility of units far off from the bond initiating the reorientation. Thus it may be possible to extend the DRIS formalism to the dynamics of larger scale motions provided that a correction in the effective isomeric transition rates is introduced to account for the above-described reduced mobility. The recently introduced mathematical method of stochastic weight matrices multiplication ${ }^{18,19}$ allows for a very systematic and computationally efficient way of incorporating this effect into the DRIS formalism. The essential tool is the adoption of a position-dependent friction coefficient, as first proposed by Paul and $\mathrm{Mazo}^{20}$ and used in the study of helix-coil transitions of polypeptides. ${ }^{21}$ The agreement between the results of Fixman and the DRIS simulations reported in the following sections serves as an a posteriori proof of the validity of the latter scheme.

In the next section, the physical and mathematical foundations underlying the DRIS model will be recapitulated with emphasis on its extension to cover intermediate regime dynamics. An advantage this analysis offers is that it is possible to evaluate the cross-correlation functions in addition to autocorrelations and to estimate the relative importance of cross-modes coupling in relaxation. Illustrative calculations and comparison with previous work will be presented in the third section, which will be followed by the discussion in the final section.

\section{Theory}

2.1. The Model and the General Approach. In general, the orientational dynamics results from the superposition of various wavelength motions. One can think of different sizes of independent kinetic units depending on the experimental time window of observation. The kinetic unit may be viewed as an ensemble of bonds whose coupled motion is essentially responsible for the experimental observation. For instance, in NMR relaxation the kinetic unit apparently consists of approximately three to five skeletal bonds while light scattering techniques probe global diffusive motions. Clearly, the apparent relaxation times increase with the size of the kinetic unit, i.e., with the number of bonds participating in a concerted motion.

A kinetic unit of $2 n+1$ bonds will be considered, in general. Figure 1 illustrates the particular case for $n=10$. The dashed lines on both sides of the unit indicate the tails. $m$ is the vector of interest whose orientational dynamics will be analyzed. It is rigidly affixed to the 
central bond of the unit. We make the following assumptions on the dynamic behavior of the unit:

(i) The tails do not take active part in the dynamics of the kinetic unit. Stated in another way, the vector $\mathbf{m}$ is dynamically uncorrelated with the bonds beyond the two ends of the kinetic unit, hence the term "independent kinetic unit".

(ii) The first two bonds of the kinetic unit are assumed to be fixed in space and determine the laboratory fixed frame $O X Y Z$, indicated in the figure. The orientational dynamics of $\mathbf{m}$ will be treated with respect to that frame.

(iii) The portion of the kinetic unit between the origin $O$ and the vector $m$ is assumed to undergo all rotameric transitions in the configurational space, while the second half of the kinetic unit is not explicitly considered in the analysis. The presence of second half of the kinetic unit necessarily slows down the motion. The retarding effect of the second half will be incorporated in the effective friction coefficient experienced by the moving bonds as will be delineated in section 2.3 .

(iv) A single transition is assumed to take place at a given time, by any bond $i(1 \leq i \leq n)$ to the left of $\mathbf{m}$, within the independent kinetic unit. A possible transition is shown by the curved arrow in the figure.

(v) An isomeric rotation over bond $i$ sets in motion the bonds located to its right. Thus the motion propagates from left to right; i.e., the mobility of the vector $\mathbf{m}$ is assumed to be imparted by the rotameric transitions of the bonds located to its left only. Actually, rotameric transitions over bonds situated to the right of $\mathbf{m}$ also contribute equally to the mobility of $\mathbf{m}$. However, neglecting their contribution is inconsequential, since the contribution from the rotating bonds to the left of $\mathbf{m}$ is fully reflected in the direction of $\mathbf{m}$, instead of being evenly distributed over both sides of the rotating bonds.

The autocorrelation of $\mathbf{m}$ as well as its cross-correlation with other vectors along the kinetic unit are of special interest. It should be noted that the chain model adopted by Fixman in direct Langevin simulations is based on less stringent assumptions than those adopted in the present study.

In parallel with the conventional picture of Markov chains of equilibrium statistics, pairwise interdependence of skeletal bonds is the basic approximation originally adopted in the DRIS formalism, to account for the contribution of short-range intramolecular energetics. ${ }^{7}$ The latter is directly responsible for the above-described internal viscosity effect. Also, the intramolecular elastic resistance that originates from nothing else than the perturbation of the configurational distribution is inherently present in this approach inasmuch as the complete probability space of accessible configurations is rigorously considered. The incorporation of the influence of the longchain connectivity opposing or damping the motion will be deferred to section 2.3 , as mentioned in assumption iii presented above.

For the case of $\nu$ isomeric states $\alpha, \beta, \gamma, \ldots$ accessible to each bond, $\nu^{2} \times \nu^{2}$ distinct types of pair conformational transitions are operative. For a given pair of skeletal bonds $(i-1, i)$, the probability of occurrence of configurations $\{\alpha \beta\}$ and $\left\{\alpha^{\prime} \beta^{\prime}\right\}$ within a time interval $\tau$ is referred to as the time-delayed joint probability for the pair and indicated as $p_{i}\left(\alpha \beta ; \alpha^{\prime} \beta^{\prime}\right)$. Single-bond time-delayed joint probabilities may be deduced from

$$
p_{i-1}\left(\alpha ; \alpha^{\prime}\right)=\sum_{\beta} \sum_{\beta^{\prime}} p_{i}\left(\alpha \beta ; \alpha^{\prime} \beta^{\prime}\right)
$$

Serial multiplication of joint probabilities following the rule

$$
\begin{gathered}
p_{n}\left(\alpha \beta \xi \delta \zeta \ldots \eta \rho ; \alpha^{\prime} \beta^{\prime} \xi^{\prime} \delta^{\prime} \zeta^{\prime} \ldots \eta^{\prime} \rho^{\prime}\right) \equiv p_{\tau}\left[\{\Phi\} ;\{\Phi\}^{\prime}\right]=p_{2}\left(\alpha \beta ; \alpha^{\prime} \beta^{\prime}\right) \times \\
\frac{p_{3}\left(\beta \xi ; \beta^{\prime} \xi^{\prime}\right)}{p_{2}\left(\beta ; \beta^{\prime}\right)} \frac{p_{4}\left(\xi \delta ; \xi^{\prime} \delta^{\prime}\right)}{p_{3}\left(\xi ; \xi^{\prime}\right)} \frac{p_{5}\left(\delta \zeta ; \delta^{\prime} \xi^{\prime}\right)}{p_{4}\left(\delta ; \delta^{\prime}\right)} \ldots \frac{p_{n}\left(\eta \rho ; \eta^{\prime} \rho^{\prime}\right)}{p_{n-1}\left(\eta ; \eta^{\prime}\right)}(2)
\end{gathered}
$$

yields the time-delayed joint probability of occurrence of configurations $\{\Phi\} \equiv\{\alpha \beta \xi \delta\} \ldots \eta \rho\}$ and $\{\Phi\}^{\prime} \equiv\left\{\alpha^{\prime} \beta^{\prime} \xi^{\prime} \delta^{\prime} \zeta^{\prime} \ldots \eta^{\prime} \rho^{\prime}\right\}$, with the given time interval $\tau$, for a sequence of $n$ bonds, ${ }^{7}$ indexed from 1 to $n$. Naturally, with the knowledge of the time-delayed joint probabilities for all of the accessible configurational transitions, complete information on the dynamics of the $n$-bond sequence is acquired. Accordingly, the ensemble average value $\langle f(\tau)\rangle$ of any time-dependent property $f\left[\{\Phi\} ;\{\Phi\}^{\prime}\right]$, associated with the configurational transition $\{\Phi\} \rightarrow\{\Phi\}$, is readily evaluated from

$$
\langle f(\tau)\rangle=\sum_{\{\Phi \mid} \sum_{\left\langle\left.\Phi\right|^{\prime}\right.} f\left[\{\Phi\} ;\{\Phi\}^{\prime}\right] p_{\tau}\left[\{\Phi\} ;\{\Phi\}^{\prime}\right]
$$

The above double summation, which becomes prohibitively time-consuming to perform as $n$ increases, may be readily computed by the matrix multiplication scheme recently developed to treat chain conformational stochastics, ${ }^{18,19}$ in analogy to the mathematical methods devised ${ }^{22}$ for the linear Ising model and commonly employed in chain statistical mechanics. ${ }^{23}$ The scheme for the evaluation of orientational correlation functions (OCF) will be summarized in the next section.

2.2. Matrix Multiplication Scheme To Evaluate OCFs. The method relies on the serial multiplication of stochastic weight matrices of the form ${ }^{18}$

$$
\mathbf{V}_{2}(\tau)=\left[\begin{array}{llll}
p(\mathrm{t} ; \mathrm{t}) & & & \\
& p\left(\mathrm{t} ; \mathrm{g}^{+}\right) & & \\
& & p\left(\mathrm{t} ; \mathrm{g}^{-}\right) & \\
& & & p\left(\mathrm{~g}^{-} ; \mathrm{g}^{-}\right)
\end{array}\right]
$$

and

$$
\mathbf{V}_{i}(\tau)=\left[\begin{array}{lll}
v_{i}(\mathrm{tt} ; \mathrm{tt}) & v_{i}\left(\mathrm{tt} ; \mathrm{tg}^{+}\right) & \ldots \\
v_{i}\left(\mathrm{tt} ; \mathrm{g}^{+} \mathrm{t}\right) & v_{i}\left(\mathrm{tt} ; \mathrm{g}^{+} \mathrm{g}^{+}\right) & \ldots \\
v_{i}\left(\mathrm{tt} ; \mathrm{g}^{-} \mathrm{t}\right) & v_{i}\left(\mathrm{tt} ; \mathrm{g}^{-} \mathbf{g}^{+}\right) & \ldots \\
v_{i}\left(\mathrm{~g}^{+} \mathrm{t} ; \mathrm{tt}\right) & v_{i}\left(\mathrm{~g}^{+} \mathrm{t} ; \mathrm{tg}^{+}\right) & \ldots \\
v_{i}\left(\mathrm{~g}^{+} \mathrm{t} ; \mathrm{g}^{+} \mathrm{t}\right) & v_{i}\left(\mathrm{~g}^{+} \mathrm{t} ; \mathrm{g}^{+} \mathrm{g}^{+}\right) & \ldots \\
v_{i}\left(\mathrm{~g}^{+} \mathrm{t} ; \mathrm{g}^{-} \mathrm{t}\right) & v_{i}\left(\mathrm{~g}^{+} \mathrm{t} ; \mathrm{g}^{-} \mathrm{g}^{+}\right) & \ldots \\
v_{i}\left(\mathrm{~g}^{-} \mathrm{t} ; \mathrm{tt}\right) & \ldots & v_{i}\left(\mathrm{~g}^{-} \mathbf{g}^{-} ; \mathrm{g}^{-} \mathrm{t}\right) \\
v_{i}\left(\mathrm{~g}^{-} \mathrm{t} ; \mathrm{g}^{+} \mathrm{t}\right) & \ldots & v_{i}\left(\mathrm{~g}^{-} \mathbf{g}^{-} ; \mathrm{g}^{-} \mathbf{g}^{+}\right) \\
v_{i}\left(\mathrm{~g}^{-} \mathrm{t} ; \mathrm{g}^{-} \mathrm{t}\right) & \ldots & v_{i}\left(\mathrm{~g}^{-} \mathbf{g}^{-} ; \mathrm{g}^{-} \mathbf{g}^{-}\right)
\end{array}\right]
$$

with

$$
v_{i}(\alpha \beta ; \gamma \zeta) \equiv p_{i}(\alpha \beta ; \gamma \zeta) / p_{i-1}(\alpha ; \gamma)
$$

and $3 \leq i \leq n$, for the case of three rotational isomeric states trans $(\mathrm{t})$, gauche ${ }^{+}\left(\mathrm{g}^{+}\right)$, and gauche $\mathrm{e}^{-}\left(\mathrm{g}^{-}\right)$accessible to each of the $n$ rotating bonds.

The case where $\langle f(\tau)\rangle$ is the orientational autocorrelation function $\langle\mathbf{m}(0) \cdot \mathbf{m}(\tau)\rangle$ associated with the abovedescribed central vector $m$ of a kinetic unit of $2 n+1$ bonds will be considered in the following. $m$ moves in correlation with the preceding $n$ bonds, as delineated in the model. The instantaneous positions of $\mathbf{m}$ are pre- 
scribed by $\{\Phi\}$ and $\{\Phi\}^{\prime}$ at time 0 and $\tau$, respectively, such that

$$
\mathbf{m}(0) \cdot \mathbf{m}(\tau)=\mathbf{T}\{\Phi\} \mathbf{m}^{\circ} \cdot \mathbf{T}\{\boldsymbol{\Phi}\}^{\prime} \mathbf{m}^{\circ}
$$

where $\mathbf{m}^{\circ}$ is the vectorial representation of $\mathbf{m}$ in the $j$ th local bond-based frame in which it is rigidly embedded and $T\{\Phi\}$ is the frame transformation operator, which depends on the configuration $\{\Phi\}$ of the $n$ bonds. The orientational autocorrelation function $\langle\mathbf{m}(0) \cdot \mathbf{m}(\tau)\rangle$ may be computed by the matrix multiplication scheme according to eqs $8-11 . .^{19}$

$$
\langle\mathbf{m}(0) \cdot \mathbf{m}(\tau)\rangle=\mathbf{m}^{\circ \mathrm{T}}\left(\mathbf{D} \otimes \mathbf{E}_{3}\right)\left(\mathbf{E}_{3} \otimes \mathrm{F}\right) \mathbf{m}^{\circ}
$$

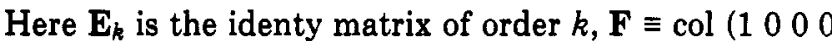
10001 ), the symbol $\otimes$ represents the direct product, the superscript $T$ indicates the transpose, and the matrix $\mathbf{D}$ is defined as

$$
\mathbf{D} \equiv \mathbf{F}^{\mathrm{T}}\left\langle\prod_{i=1}^{n}\left[\mathbf{T}_{i}(0) \otimes \mathbf{T}_{i}(\tau)\right]\right\rangle
$$

in which

$$
\left\langle\prod_{i=1}^{n}\left[\mathbf{T}_{i}(0) \otimes \mathbf{T}_{i}(\tau)\right]\right\rangle
$$

is found from ${ }^{24}$

$$
\begin{aligned}
& \left\langle\prod_{i=1}^{n}\left[\mathbf{T}_{i}(0) \otimes \mathrm{T}_{i}(\tau)\right]\right\rangle= \\
& \quad\left(\mathbf{J}^{\mathrm{T}} \otimes \mathbf{E}_{9}\right) \prod_{i=2}^{n}\left[\left(\mathbf{V}_{i}(\tau) \otimes \mathbf{E}_{9}\right)\left\|\mathbf{T}_{i}(0) \otimes \mathbf{T}_{i}(\tau)\right\|\right]\left(\mathbf{J} \otimes \mathbf{E}_{9}\right)
\end{aligned}
$$

Here $\mathbf{J} \equiv \operatorname{col}\left(\begin{array}{llll}1 & 1 & \ldots . & 1\end{array}\right)$ and $\mathbf{T}_{i}(\tau)$ is the conventional orthogonal matrix transforming vectorial or tensorial quantities rigidly fixed in the bond-based local coordinate system $i+1$ into their representations in frame $i$, on the basis of the rotational isomeric state of bond $i$ at time $\tau . .^{12}$ Thus, $\mathbf{T}\{\Phi\}^{\prime}$ equates to $\prod_{i=2}^{n} \mathbf{T}_{i}(\tau)$. A similar relationship in terms of $\mathbf{T}_{i}(0)$ holds to $\mathbf{T}\{\boldsymbol{\Phi}\}$. It is noted that the first bond of the kinetic unit is not considered. The latter prescribes the absolute orientation of the kinetic unit, which does not affect the internal orientational correlations. $\left\|T_{i}(0) \otimes T_{i}(\tau)\right\|$ is the diagonal supermatrix of the form ${ }^{19}$

$$
\left\|\mathbf{T}_{i}(0) \otimes \mathbf{T}_{i}(\tau)\right\|=\left[\begin{array}{llll}
\mathbf{T}_{\mathrm{t}} \otimes \mathbf{T}_{\mathrm{t}} & & & \\
& \mathbf{T}_{\mathrm{t}} \otimes \mathbf{T}_{\mathrm{g}^{+}} & & \\
& & \ldots & \mathbf{T}_{\mathrm{g}} \otimes \mathbf{T}_{\mathrm{g}}
\end{array}\right]
$$

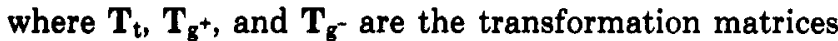
where the torsional angles for the $t, g^{+}$, and $g^{-}$states of bond $i$ are inserted.

The above formulation, which has been developed for the evaluation of orientational autocorrelation functions, may be readily extended to cross-correlations between distinct vectors along the chain. Let us consider, for instance the cross-correlation between the vectors $u$ and $v$, situated respectively at the $i$ th and $j$ th bond-based references frames of a given kinetic unit. Let us take $j>$ $i$. The cross-correlation between $\mathbf{u}$ and $\mathbf{v},\langle\mathbf{u}(0) \cdot \mathbf{v}(\tau)\rangle$, may be evaluated with the same mathematical schemes as the one presented above for the autocorrelation of $\mathbf{m}$, provided that the transformation matrices $\mathbf{T}_{p}(0)$ corresponding to the local frames in the range $i \leq p \leq j-1$ are set equal to the identity matrix of order 3 .
So far we presented the methodology for an efficient computation of orientational correlations. Clearly, correspondence with real chain behavior relies on the assignment of realistic transition probabilities for the bonds in the kinetic unit. The determination of the effective rotameric transition rates will be treated in the next subsection.

2.3. Determination of Time-Delayed Joint Probabilities $p_{i}(\alpha \beta ; \zeta \eta)$. Two major factors are considered in the evaluation of the stochastic weights associated with the isomeric transitions of a given pair of bonds: (i) The intramolecular conformational energy barrier to be surmounted when effectuating the rotation and (ii) the frictional resistance increasing with the size of the reorienting unit accompanying a given bond rotation. Clearly, those effects are of short-range and long-range character, respectively. The former may be estimated from the considerations of two-dimensional energy maps, constructed as a function of two consecutive torsional angles; this yields information on the heights of the saddles whose relative values with respect to isomeric minima will be used as the activation energies in the classical Arrheniustype expressions for the rate constants. The second effect will be treated through adoption of position-dependent friction coefficients in the front factor of rate constants, as will be presented below.

In general, for a given time $\tau$ the time-delayed joint probabilities $p_{i}(\alpha \beta ; \zeta \eta)$ are related to the transition or conditional probabilities $c_{i}(\alpha \beta / \zeta \eta)$ by the relationship

$$
p_{i}(\alpha \beta ; \zeta \eta)=c_{i}(\zeta \eta / \alpha \beta) p_{i}{ }^{\circ}(\alpha \beta)
$$

where $p_{i}{ }^{\circ}(\alpha \beta)$ is the equilibrium probability of state $\{\alpha \beta\}$ for bonds $(i-1, i)$. The latter may be directly determined from the Boltzmann weight of the corresponding state in the ensemble of $\nu^{2}$ isomeric pairs. The conditional probability $c_{i}(\zeta \eta / \alpha \beta)$ for the indicated transition is an element of the $\nu^{2} \times \nu^{2}$ transition probability matrix $\mathbf{C}_{i}(\tau)$ for the interdependent pairs of bonds $(i-1, i)$. The latter reads $^{6-8}$

$$
\mathbf{C}_{i}(\tau)=\exp \left\{-\mathbf{A}_{i} \tau\right\}=\mathbf{B}_{i} \exp \left\{-\Lambda_{i} \tau\right\} \mathbf{B}_{i}^{-1}
$$

Here $\mathbf{A}_{i}$ is the transition rate matrix of order $\nu^{2}$ governing the kinetics of pair transitions, $B_{i}$ and $\Lambda_{i}$ are the respective matrices of eigenvectors and eigenvalues resulting from the diagonalization $\mathbf{A}_{i}=\mathbf{B}_{i} \boldsymbol{\Lambda}_{i} \mathbf{B}_{i}^{-1}$ of $\mathbf{A}_{i}$. The off-diagonal elements of $\mathbf{A}_{i}$ represent the rate constants associated with the transitions between the isomeric states $\{t t\},\left\{\operatorname{tg}^{+}\right\}, \ldots$, $\left\{\mathrm{g}^{-} \mathrm{g}^{-}\right\}$, which are numbered from 1 to 9 , such that the element $A_{I J}$ refers to the passage from the $J$ th to the $I$ th state. In conformity with the principle of detailed balance, each column in $\mathbf{A}_{i}$ sums up to zero, which determines the value of the diagonal elements. Explicit expressions for $\mathbf{A}_{i}$ may be found from refs 6-8. The rate constants $r_{i}$ therein are given by Kramers' high-friction limit expression as

$$
r_{i}=\frac{\left(\gamma^{*} \gamma\right)^{1 / 2}}{2 \pi \zeta_{i, \text { eff }}} \exp \left\{-E_{\text {act }} / R T\right\}
$$

where $\gamma$ and $\gamma^{*}$ refer to the curvature of the energy path at the minimum and saddle point, respectively, $E_{\text {act }}$ is the activation energy for the specific transition, and $\zeta_{i, \text { eff }}$ is the effective friction coefficient for bond $i$. For the case where $(k-i)$ bonds, including the vector $\mathbf{m}$ affixed to the bond $n$, are set in motion upon the rotameric transition of bond $i, \zeta_{i, \text { eff }}$ appearing in eq 14 may be expressed ${ }^{11,25}$ as 


$$
\zeta_{i, \text { eff }}=\zeta^{0} \sum_{p=i+1}^{k}\left\langle s_{i p}{ }^{2}\right\rangle=\zeta^{0}\left[\sum_{p=i+1}^{n}\left\langle s_{i p}{ }^{2}\right\rangle+\sum_{p=n+1}^{k}\left\langle s_{i p}{ }^{2}\right\rangle\right]
$$

where $\zeta^{0}$ is a constant of proportionality and $s_{i p}$ is the separation of the $p$ th atom from the axis of rotation defined by the bond $i$, which undergoes the isomeric transition. The brackets indicate the average over all configurations accessible to the moving units. In general, the number $k$ $-i$ of bonds set in motion upon the rotameric transition over bond $i$ is not uniquely defined and depends on the individual configurations of the $k-i$ bonds. Consequently, the location of the vector $m$ relative to bonds $i$ and $k$ varies from one configuration to the other. However, on the average, inasmuch as $\mathbf{m}$ is confined to be at the center of the independent kinetic unit, bonds at both sides of $\mathbf{m}$ may be assumed to experience same frictional resistance. Consequently the effective friction coefficient reduces to

$$
\zeta_{i, \mathrm{eff}}=2 \zeta^{0} \sum_{p=i+1}^{n}\left\langle s_{i p}{ }^{2}\right\rangle
$$

which, from moment of inertia considerations for a random coil, ${ }^{21}$ may be expressed by the proportionality $(n-i)^{2}$. Also, in view of the lack of precise quantitative knowledge on $\zeta^{\circ}, \gamma$, and $\gamma^{*}$, those parameters may be combined as a single proportionality constant $A_{\mathrm{o}}$ and the expression for the effective rate constant becomes

$$
r_{i}=A_{0}(n-i)^{-2} \exp \left\{-E_{\text {act }} / R T\right\}
$$

The above equation deserves special attention. The exponential part arises from the specific chain conformational energetics whereas the term $(n-i)^{-2}$ may be viewed as a property common to all chainlike structures and responsible for the slowing down and/or damping of the propagation of reorientation away from the rotating bond. Accordingly, $r_{i}$ represents the effective rate constant associated with the motion of the $n$th bond as a result of the isomeric transition of bond $i$.

According to eq 16 , a position-dependent rate is assigned to skeletal bonds, depending on their location relative to the investigated bond $n$ in the kinetic unit. Clearly, the latter is identified by the vector $m$ whose orientational behavior is investigated. Its dynamic behavior is then accepted to be characteristic of the kinetic unit where it belongs. $n$ will be varied in the range $n \leq 100$, for an understanding of the variation of the apparent relaxation times with the size of kinetic units.

2.4. Orientational Correlation Times. A quantitative measure of the orientational correlations between different vectorial quantities rigidly embedded in a given kinetic segment is the associated correlation time, which may be readily determined from the area enclosed by the normalized time decay curves of the OCFs. Accordingly, for a pair of bond vectors $\mathbf{l}_{i}$ and $\mathbf{l}_{j}$ the orientational correlation time $\tau_{i j}$ equates to

$$
\tau_{i j}=\int_{0}^{\infty} \frac{\left\langle\mathbf{l}_{i}(0) \cdot \mathbf{l}_{j}(\tau)\right\rangle-\left\langle\mathbf{l}_{i}(0) \cdot \mathbf{l}_{j}(\infty)\right\rangle}{\left\langle\mathbf{l}_{i}(0) \cdot \mathbf{l}_{j}(0)\right\rangle-\left\langle\mathbf{l}_{i}(0) \cdot \mathbf{l}_{j}(\infty)\right\rangle} \mathrm{d} \tau
$$

Using the identities $\left\langle\mathbf{l}_{i}(0) \cdot \mathbf{l}_{j}(\infty)\right\rangle=\left\langle\mathbf{l}_{i}(0)\right\rangle \cdot\left\langle\mathbf{l}_{j}(\infty)\right\rangle=\left\langle\mathbf{l}_{i}\right\rangle \cdot\left\langle\mathbf{l}_{j}\right\rangle$ and $\left\langle\mathbf{l}_{i}(\mathbf{0}) \cdot \mathbf{l}_{j}(0)\right\rangle=\left\langle\mathbf{l}_{i} \cdot \mathbf{l}_{j}\right\rangle$, where the time arguments of static properties are omitted for brevity, eq 17 may be rewritten as

$$
\tau_{i j}=\left(\left\langle\mathbf{1}_{i} \cdot \mathbf{1}_{j}\right\rangle-\left\langle\mathbf{1}_{i}\right\rangle \cdot\left\langle\mathbf{1}_{j}\right\rangle\right)^{-1} \tilde{\tau}_{i j}
$$

where the unnormalized correlation time $\tilde{\tau}_{i j}$ is defined by

$$
\tilde{\tau}_{i j} \equiv \int_{0}^{\infty}\left(\left\langle\mathbf{l}_{i}(0) \cdot \mathbf{l}_{j}(\tau)\right\rangle-\left\langle\mathbf{1}_{i} \mathbf{l}_{j}\right\rangle\right) \mathrm{d} \tau
$$

For a given segment of $n$ bonds, $\tau_{i j}$ associated with any pair of bonds $1 \leq i \leq j \leq n$ may be conveniently written as the elements of a correlation time matrix $\tau$. This is a symmetric matrix of order $n$, with the elements on the diagonal representing the autocorrelation times and the off-diagonal elements $\tau_{i j}$ with $i \neq j$, the cross-correlation time between bond vectors separated by $(j-i)$ bonds. Alternately, the reciprocal of $\tau_{i j}$ may be viewed as the relaxation rates associated with different order orientational correlations.

\section{Calculations}

3.1. The Chain Parameters. A hypothetical polyethylene-like chain with perfect tetrahedral geometry will be considered. Three states $t, \mathrm{~g}^{+}$, and $\mathrm{g}^{-}$with respective torsional angles $0^{\circ}, 120^{\circ}$, and $-120^{\circ}$ and energies $0,0.5$, and $0.5 \mathrm{kcal} / \mathrm{mol}$ will be assigned to each skeletal bond. The $\mathbf{g}^{+} \mathrm{g}^{-}$and $\mathrm{g}^{-} \mathrm{g}^{+}$states leading to a pentane effect will be assigned an extra second-order interaction energy of $2.0 \mathrm{kcal} / \mathrm{mol}$, in analogy with conventional treatments of polyethylene chains. No second-order interaction energy will be present in the other isomeric states and their energies will be found from simple addition of the single bond conformational energies. Thus bond interdependence comes into play only through occurrence of $\mathrm{g}^{+} \mathrm{g}^{-}$or $\mathrm{g}^{-} \mathrm{g}^{+}$states. Heights of the barriers surrounding the isomeric tt minima in two-dimensional energy maps will be taken as $3.5 \mathrm{kcal} / \mathrm{mol}$. The passages $\mathrm{g}^{+} \leftrightarrow \mathrm{g}^{-}$are precluded and the escapes from $\mathrm{g}^{ \pm} \mathrm{g}^{ \pm}$and $\mathrm{g}^{ \pm} \mathrm{g}^{\mp}$ states necessitate activation energies of 3.0 and $0.5 \mathrm{kcal} / \mathrm{mol}$, respectively. The proportionality constant $A_{0}$ in the rate constants is taken as $2.77 \times 10^{11} / \mathrm{s}$.

3.2. Evaluation of Static and Dynamic Orientational Correlations. The orientational correlation functions $\left\langle\mathbf{l}_{i}(0) \cdot \mathbf{l}_{j}(\tau)\right\rangle$ have been evaluated following the scheme outlined in section 2.2. For that purpose as a first step, time-delayed joint probabilities are determined by the approach of section 2.3 and inserted into the stochastic weight matrices, which constitute the basis of the matrix multiplication scheme.

For the particular case of autocorrelations, both the subscripts $i$ and $j$ in $\left\langle\mathbf{l}_{i}(0) \cdot \mathbf{l}_{j}(\tau)\right\rangle$ equate to $n$ and $\mathbf{m}^{\circ}$ in eq 8 is replaced by the unit vector $\left(\begin{array}{lll}1 & 0 & 0\end{array}\right)^{\mathrm{T}}$, representing the investigated bond in its local frame. Direct application of eqs 8-11 yields the autocorrelation function at a given time. The complete time dependence is obtained by repeating the procedure for several choices of $\tau$. The resulting time decay curve is numerically integrated following eq 17 to determine the corresponding correlation time $\tau$. The upper curve in Figure 2 displays the reciprocal correlation times $1 / \tau$ computed for kinetic segments of various sizes, as indicated by the abscissa $1 / i$. Thus, $1 / \tau$ is representative of the relaxation rate of a skeletal bond whose orientational motion is coupled to the preceding $i$ bonds. A gradual increase in correlation time is predicted with the increase in the number of bonds that cooperatively participate in the relaxation.

Likewise, cross-correlations are found by applying the same procedure to $1_{i}(0)$ and $l_{j}(\tau)$, where $j \neq i$. For that purpose, $j$ is equated to $n$ and the scheme of eqs 8-11 is followed with the adoption of identity matrices instead of $\mathbf{T}_{p}(0)$ in the range $i \leq p \leq j-1$. The family of curves in Figure 2 displays the correlation times associated with $\left\langle\mathbf{l}_{i}(0) \cdot \mathbf{l}_{j}(\tau)\right\rangle$ as a function of $1 / i$, for various $(j-i)$, as indicated in the caption. It is worth noting that the values of $\left\langle\mathbf{l}_{i}(0) \cdot \mathbf{l}_{j}(\tau)\right\rangle$ depend on the locations of $i$ and $j$ on the 


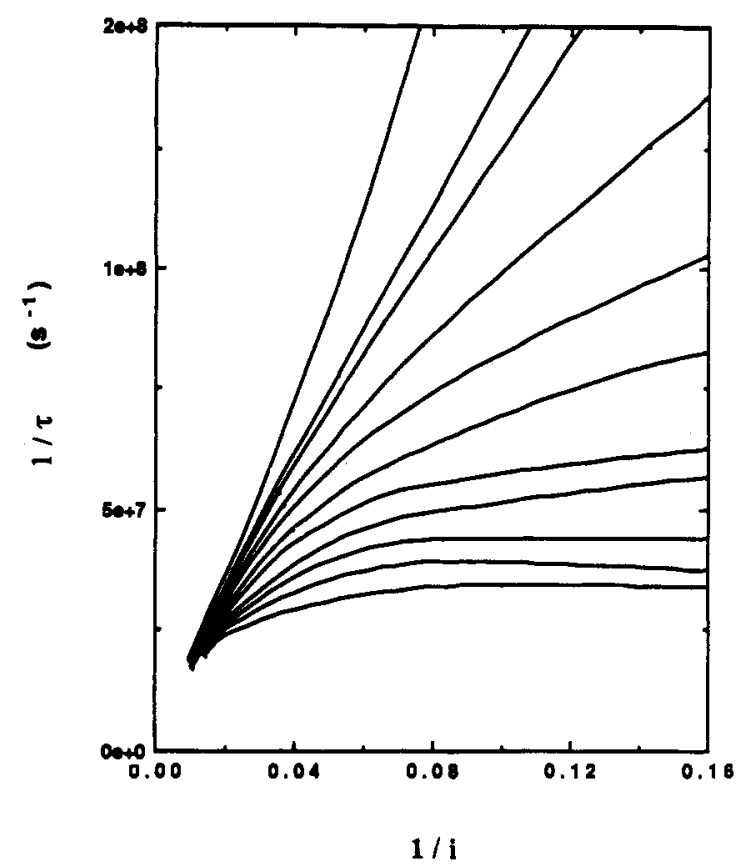

Figure 2. Dependence of reciprocal correlation times $1 / \tau$ on the position $i$ of the investigated bond. The uppermost curve corresponds to the autocorrelation $\left\langle\mathbf{l}_{i}(0) \cdot \mathbf{l}_{i}(\tau)\right\rangle$. The remaining curves represent $1 / \tau$ associated with $\left\langle\mathbf{l}_{i}(0) \cdot \mathbf{l}_{i+k}(\tau)\right\rangle$ in the order of increasing values of $k$ from 1 to 10 , such that the lowest curve corresponds to $k=10$.

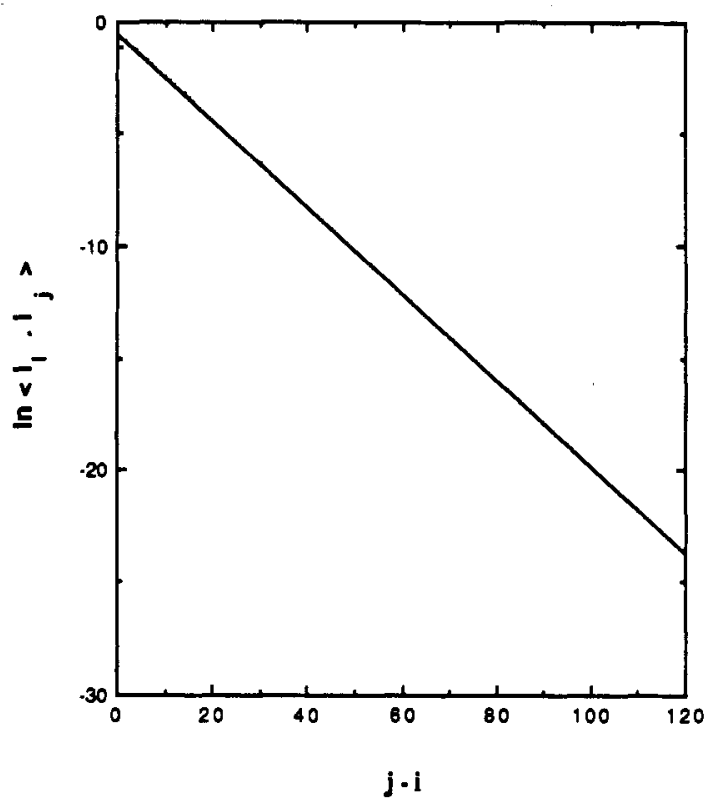

Figure 3. Loss of the static orientational correlation $\left(\mathbf{l}_{i} \cdot \mathbf{l}_{j}\right)$ with increasing separation $(j-i)$ of the bond vectors. The exponential decrease with $(j-i)$ is clearly apparent from the straight line resulting from the plot of $\left\langle\mathbf{l}_{i} \cdot \mathbf{l}_{j}\right\rangle$ versus $(j-i)$.

kinetic unit as well as on $j-i$. In other words, the correlation functions differ whether $j$ is to the right or left of $i$ with respect to the origin. The differences that arise due to short-chain effect are not appreciable, however, and only the results of the case where $j>i$ are reported in this work.

For illustrative purposes, the two static properties $\left\langle\mathbf{l}_{i} \cdot \mathbf{l}_{j}\right\rangle$ and $\left\langle\mathbf{l}_{i}\right\rangle \cdot\left(\mathbf{l}_{j}\right\rangle$ found from the intercept and the asymptotic limits of the $\left\langle\mathbf{l}_{i}(0) \cdot \mathbf{l}_{j}(\tau)\right\rangle$ versus $\tau$ curves are plotted in Figures 3 and 4, respectively. The former depends on the relative separation $j-i$ of bonds, regardless of their location in the kinetic unit. For clarity, the natural logarithm of $\left\langle\mathbf{l}_{i} \cdot \mathbf{1}_{j}\right\rangle$ is plotted against $(j-i)$. It is observed from Figure

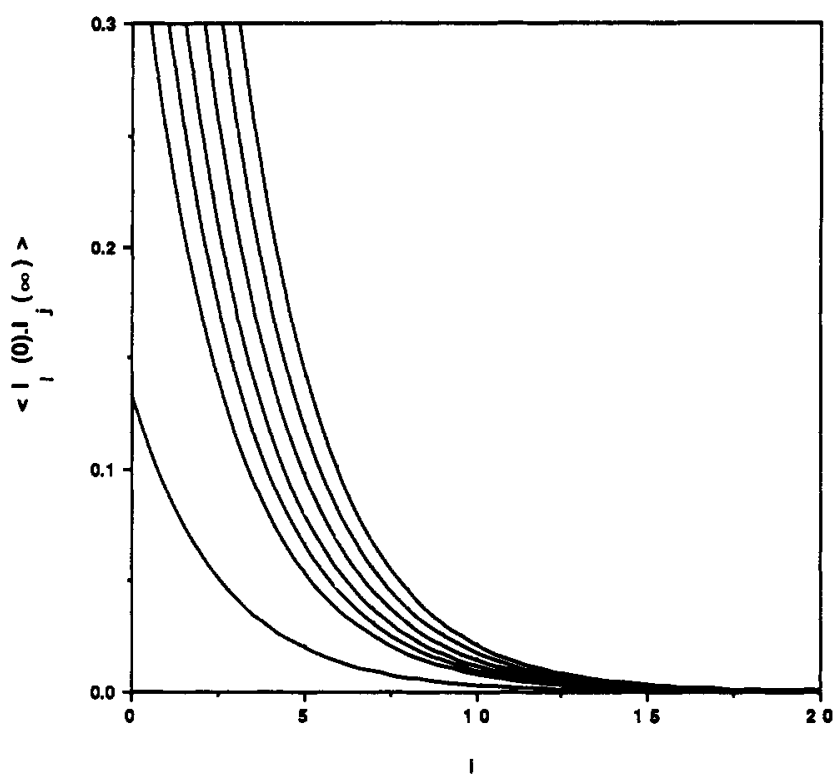

Figure 4. Dependence of infinite time cross-correlation function $\left\langle\mathbf{l}_{i}(0) \cdot \mathbf{l}_{j}(\infty)\right\rangle$ on the position $i$ of the investigated bond from the fixed end of the kinetic unit. A family of curves result from the various choices of the bond index $j$. $j$ is equal to $i+k$, with $k$ $=0,1,2,3,4,5$, and 10 in proceeding from right to left.

3 that the static orientational correlations $\left\langle\mathbf{1}_{i} \cdot \mathbf{l}_{j}\right\rangle$ are exponentially lost as the spacing of the bonds increases. The infinite time correlations $\left\langle\mathbf{l}_{i}\right\rangle \cdot\left\langle\mathbf{l}_{j}\right\rangle$ are influenced by the position of the investigated bonds with respect to the fixed ends of the kinetic unit as shown by the family of curves in Figure 4. Those correlations are found to be negligibly small beyond $\sim 15$ intermediate bonds between $l_{i}$ and $l_{j}$. The curves are plotted for several values of $j$ indices, as indicated in the corresponding figure caption.

3.3. Results in Terms of Relaxational Modes. For Gaussian model chains of $n$ bonds, the passage to normal coordinates and the corresponding independent relaxational modes is carried out through the transformation ${ }^{13,26}$

$$
\mathbf{q}=\mathbf{Q} \mathbf{l}
$$

where $l$ is the column matrix of the bond vectors $\mathbf{l}_{i}$ and $q$ is the column matrix of the normal modes $q_{i}$, with $1 \leq$ $i \leq n$. $\mathbf{Q}$ is the symmetric $n \times n$ transformation matrix with the element $Q_{i i}$ operating between the $i$ th bond vector $\mathbf{l}_{i}$ and $k$ th mode vector $\mathbf{q}_{k}$ according to

$$
Q_{k i}=\left[\frac{2}{n+1}\right]^{1 / 2} \sin \left[\frac{i k \pi}{n+1}\right]
$$

The mode correlation functions $\left\langle\mathbf{q}_{i}(0) \cdot \mathbf{q}_{j}(\tau)\right\rangle$ may be expressed, using eq 20 , as

$$
\left\langle\mathbf{q}_{i}(0) \cdot \mathbf{q}_{j}(\tau)\right\rangle=\sum_{k=1}^{n} \sum_{m=1}^{n}\left[Q_{k i} Q_{m j}\left\langle\mathbf{l}_{k}(0) \cdot \mathbf{l}_{m}(\tau)\right\rangle\right]
$$

For a system with independent modes as in the Rouse model, the cross-correlations $\left\langle\mathbf{q}_{i}(0) \cdot \mathbf{q}_{j}(t)\right\rangle$, with $i \neq j$, equate to zero. Fixman's simulations of polymer dynamics ${ }^{13}$ suggest that the contribution of cross-correlations between normal modes is of secondary importance in local relaxational processes and that cross-mode coupling may be neglected as a first-order approximation. Those inferences are drawn from the time decays of the endto-end vector and "perpendicular" dipole vector autocorrelation functions, in hypothetical short chains with $90^{\circ}$ bond angles and 3-fold symmetric rotational potentials. Also, the analysis reveals that an extremely small fraction of relaxational modes have Gaussian rates and the majority of modes exhibit a fixed rate. Mode correlation functions 


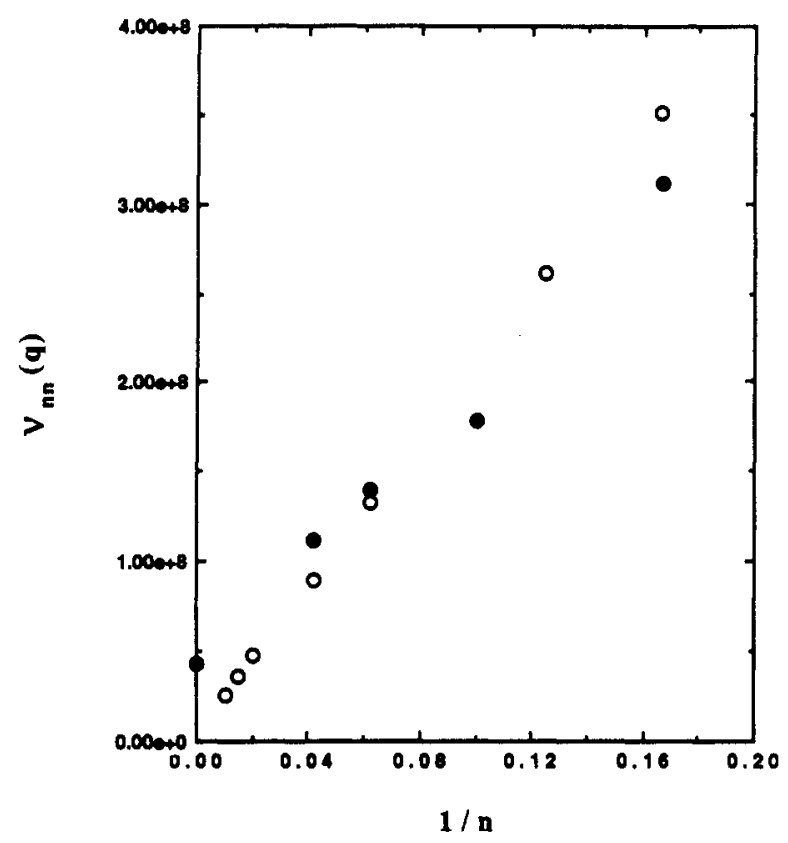

Figure 5. Dependence of the terminal normal mode relaxation rate $\nu_{n n}(q)$ on the number $n$ of bonds participating in cooperative relaxation process. The results obtained in the present study for various $n$ up to $n=150$ are represented by the empty circles. The filled circles follow from the simulations of Fixman, ${ }^{13}$ rescaled for comparative purposes. An approximately linear dependence on $n$ is predicted in both approaches. In the limit of an infinitely long chain, the least-square linear fit of the results of the present work yields a rate of $0.5 \times 10^{7} / \mathrm{s}$.

will be analyzed in the following to assess the validity of those findings. It is noted that in the present work local segments of polymer chains are treated whereas finite chains are considered in Fixman's simulations. Inasmuch as conformational relaxation in both cases results from the collective contribution of $n$ coupled bonds, it seems interesting to compare results from both approaches insofar as the qualitative dependence on $n$ and the frequency distribution of various relaxational modes are concerned.

In parallel with the well-established definition for correlation times given above, it is possible to define rates of relaxational modes $\nu_{i j}(\mathbf{q})$ as

$$
\begin{aligned}
\nu_{i j}(\mathbf{q}) \equiv\left[\boldsymbol{r}_{i j}(\mathbf{q})\right]^{-1} \equiv \\
\quad\left[\int_{0}^{\infty} \frac{\left\langle\mathbf{q}_{i}(0) \cdot \mathbf{q}_{j}(\tau)\right\rangle-\left\langle\mathbf{q}_{i}(0) \cdot \mathbf{q}_{j}(\infty)\right\rangle}{\left\langle\mathbf{q}_{i}(0) \cdot \mathbf{q}_{j}(0)\right\rangle-\left\langle\mathbf{q}_{i}(\mathbf{0}) \cdot \mathbf{q}_{j}(\infty)\right\rangle} \mathrm{d} \tau\right]^{-1}
\end{aligned}
$$

Using eqs 22 and 19, eq 23 may be rewritten as

$$
\nu_{i j}(\mathbf{q})=\tilde{\nu}_{i j}(\mathbf{q}) \sum_{k=1}^{n} \sum_{m=1}^{n} Q_{k i} Q_{m j}\left[\left\langle\mathbf{1}_{k} \cdot \mathbf{1}_{m}\right\rangle-\left\langle\mathbf{1}_{k}\right\rangle \cdot\left\langle\mathbf{1}_{m}\right\rangle\right]
$$

where

$$
\tilde{\nu}_{i j}(\mathbf{q}) \equiv\left[\sum_{k=1}^{n} \sum_{m=1}^{n}\left[Q_{k i} Q_{m j} \bar{\tau}_{i j}\right]\right]^{-1}
$$

Thus, the correlation times computed in the preceding section are readily inserted into eqs 24 and 25 to obtain the rates of relaxational modes.

Results from calculations are shown in Figures 5-7. In Figure 5, the rates $\nu_{n n}(q)$ associated with the terminal mode autocorrelation function $\left\langle\mathbf{q}_{n}(0) \cdot \mathbf{q}_{n}(\tau)\right\rangle$ are shown by the empty circles, for several size kinetic units characterized by the number $n$ of bonds participating in the motion. The filled circles represent results from Fixman's simulations, obtained with a 3-fold symmetric barrier of $4 k T$,

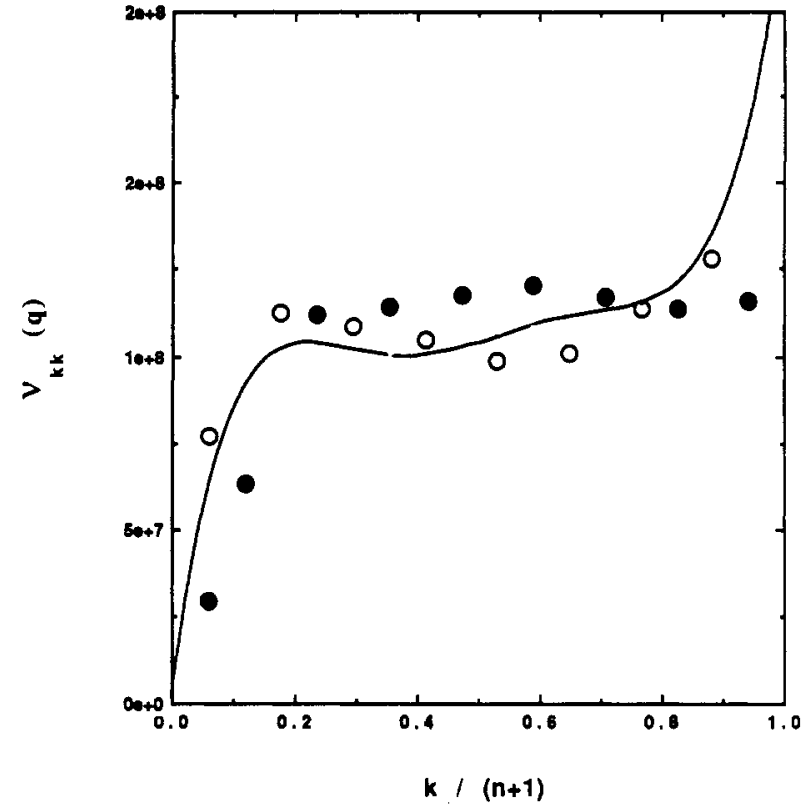

Figure 6. Dispersion of normal relaxational modes for a segment of $n=16$ coupled bonds. The rate of the $k$ th relarational mode $\nu_{k k}(q)$ is found from eqs 24 and 25 in which the unnormalized correlation time defined by eq 19 is inserted. The empty circles are computed in the present work. Filled circles are from the work of Fixman. Except for one or two slowest modes, a plateau value is observed for all $\mathbf{k}$, in both treatments. See the caption to Figure 8 for the curve drawn between the points.

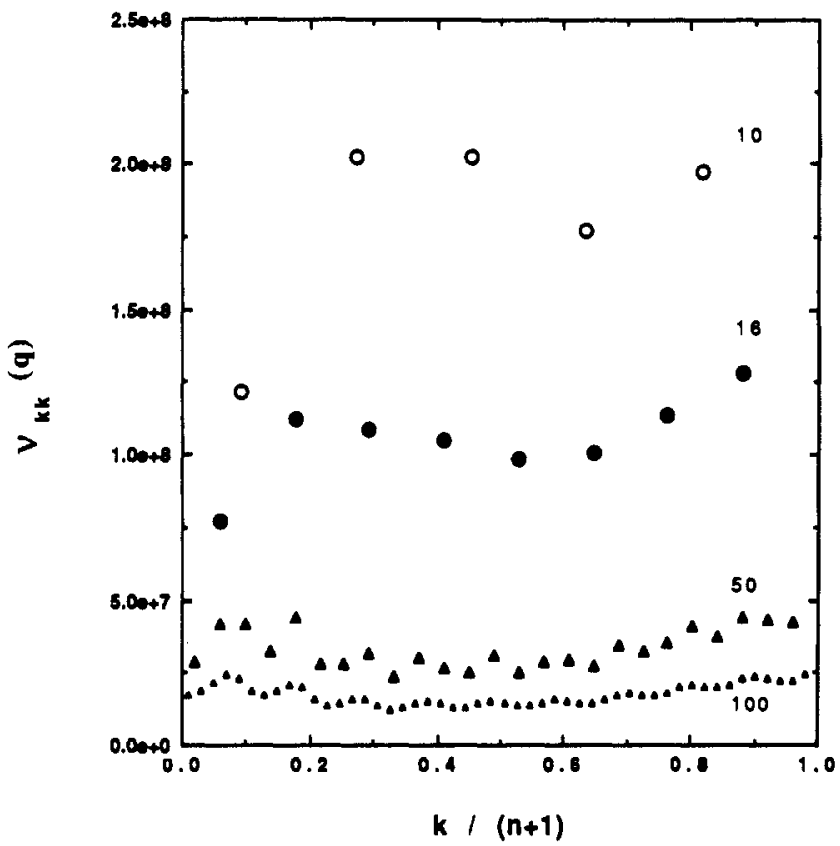

Figure 7. Dispersion of normal relaxational modes for various size kinetic units. The results are obtained for $n=10(0), 16(0)$, $50(\Delta)$, and $100(\Delta)$.

which are rescaled for a comparative analysis. The dependence of the terminal mode rate on the length of the chain segments is found to be approximately linear in conformity with Fixman's predictions. In the limit as $n$ $\rightarrow \infty$, the rate approaches a finite value equal to $5 \times 10^{6} / \mathrm{s}$, from a least-squares fit of the results.

The dependence of rates on mode number is shown in Figures 6 and 7. Figure 6 displays the results for $n=16$ obtained in the present approach (empty circles) and Fixman's work (filled circles). Similar qualitative behavior is seen in both sets of results despite the differences in the model chains and stochastic methods. The majority of 


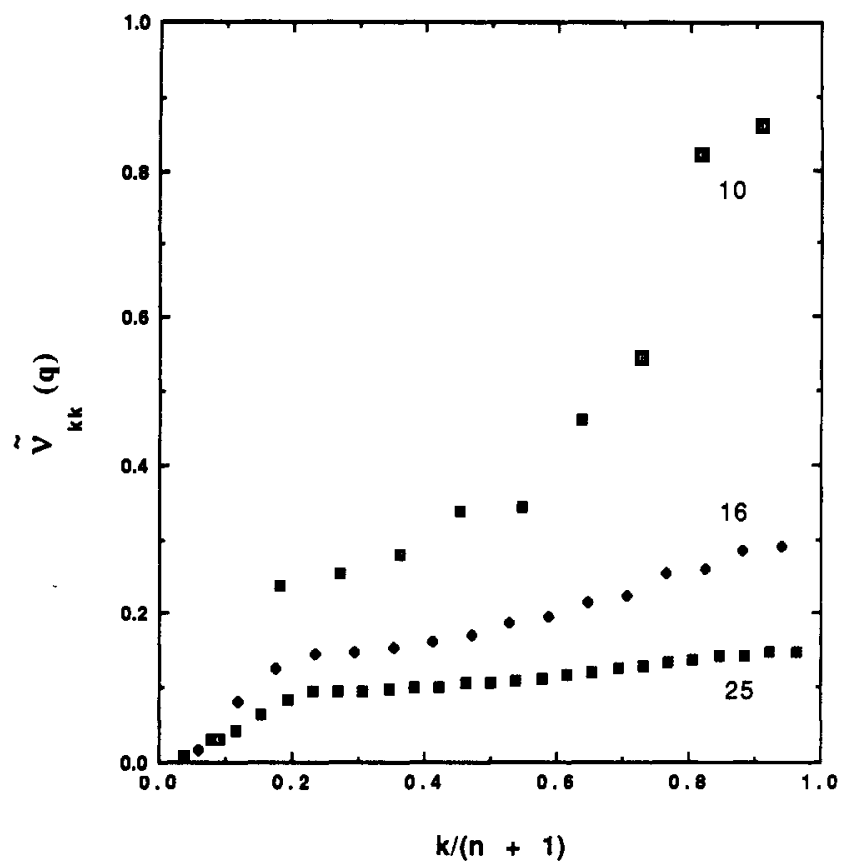

Figure 8. Unnormalized rates $\bar{\nu}_{k k}(q)$ of the exactly orthogonal relaxational modes versus $k /(n+1)$. The normalization of $\tilde{\nu}_{k k}(\mathbf{q})$ using eq 24 yields the curve displayed in Figure 6.

the modes exhibit a more or less fixed rate, except for a few slowest modes. Similar results obtained for $n=10$, 16,50 , and 100 are shown in Figure 7 for a better understanding of the dependence on chain length. The plateau value in the relaxation rates becomes even more apparent with the increase in $n$. For clarity only modes with odd $k$ values are shown in the figures.

It is noted that the above representation of chain behavior in terms of normal modes determined from the transformation of eq 22 is only approximate. The crosscorrelations do not vanish as confirmed by the nonzero values presently obtained for $\nu_{i j}(\mathbf{q})$, when $i \neq j$. On the other hand, it is possible to find a transformation matrix $\mathbf{S}$ that numerically diagonalizes the matrix $\tilde{\tau}$ of unnormalized relaxation rates, so as to yield the diagonal matrix $\tilde{\nu}$ of the unnormalized rates according to

$$
\tilde{\nu}=\left[\mathbf{S}^{\mathrm{T}} \tilde{\tau} \mathbf{S}\right]^{-1}
$$

The elements of $\ddot{\nu}$ furnish important information on the dispersion of the $n$ uncorrelated modes contributing to relaxation. They are shown in Figure 8 , for the cases $n$ $=10,16$, and 24. A smoother change with mode number is discerned in the slow relaxation regime compared to Figure 7 although the plateau behavior is still preserved over a wide range of relaxation spectrum.

The normalization of the $\bar{\nu}_{k k}(\mathbf{q})$ values obtained from eq 26 , by using eq 24, yields the curve displayed in Figure 6 , in close agreement with the approximate approach of eqs 24 and 25 . This validates the use of eqs 24 and 25 for an approximate passage to the mode domain.

\section{Discussion and Conclusions}

The results obtained in the present study are based on a systematic evaluation of the time-delayed joint probabilities of a sequence of bonds in a long chain. The assumption of a position-dependent friction coefficient is central to the formulation. Its incorporation into the formulation is not rigorously derived but is only postulated, following previous treatments. ${ }^{20,21,25}$ Yet the basic results are in striking agreement with predictions from direct Langevin simulations. Those results are mainly (i) the linear dependence of apparent relaxation times on the number $n$ of bonds participating in relaxation and (ii) the existence of a substantially large number of high-frequency modes forming a pronounced plateau. The agreement may be taken as a support of the dynamic rotational isomeric state model and its extension to longer sequences. It would be desirable to establish a more rigorous connection between the present model and the general Langevin treatment. The relationship would follow by properly expanding the dominating master equation into the Taylor series, under the smoothness conditions imposed by the position-dependent friction coefficient, and comparing with the conventional equations of chain dynamics. At the present we are not able to derive mathematically rigorous analogies, however.

According to the DRIS model, the dynamics of a sequence originates from single-bond transitions taking place over time scales of about $10^{-11} \mathrm{~s}$. Experimental and theoretical arguments in favor of activation energies of the order of those involved in single-bond transitions are now well established in the literature. ${ }^{27-29}$ The longer time dynamics of moving sequences of the present model is the accumulated effect of such single-bond transitions. The present stochastic treatment directly determines the evolution of the collective dynamics of the chain. Whether it is the best way of extrapolation to longer times may only be established by comparison with several other simulation techniques. It should be noted at this point that the present matrix generation technique offers distinct computational advantages. For example, the joint probabilities for all transitions for a chain of several hundred bonds may be carried out without any serious time or space requirements in an ordinary computer, while only very few trajectories of a chain of about hundred bonds may be generated with molecular dynamics or Brownian simulations. Furthermore, the real chemical structure of the chain may very easily be incorporated into the present calculation scheme. The effort required is equivalent to that of the well-established RIS scheme of chain statistics. Because of such advantages, it was possible for us to evaluate, for the first time, the time-dependent crosscorrelations of bond vectors in full detail and obtain an estimate of the range of correlations in the polyethylene chain. Thus, it becomes possible to investigate, rigorously, some long-standing important problems of dynamic correlations such as the validity of resolving the bond autocorrelation function into a product of an internal and an external orientational autocorrelation function. ${ }^{30}$

It should be mentioned that the chain connectivity effect inhibiting large-scale motions of the tails is not rigorously considered but is treated through a mean-field formalism based on the adoption of a dynamically independent kinetic unit moving symmetrically with respect to a central bond in which the vector of investigation is embedded. The two portions of the kinetic unit are assembled to undergo cooperative transitions so as to localize the motion within the kinetic unit. Accordingly the first half of the kinetic unit is assumed to undergo any transition, provided that the experienced friction coefficient is rescaled to account for the drag exerted on the overall kinetic unit. This approximation affects the absolute rates of relaxation without altering the frequency distribution of relaxational modes, which is the subject of interest in the present work.

It is interesting to note that the molecular geometry adopted by Fixman is much simpler than the real and detailed structure of polyethylene assumed in this study. Also, the bond transitions are more detailed in the present model and include neighbor interdependence effects and 
exact locations and energies of saddle points through which the transitions take place. Such details do not seem to strongly influence qualitative results such as the scaling of rates with the length of the sequence and the global distribution of rates for a given sequence, which by their very nature are of generic origin. It should be noted, however, that such details are of major importance in comparing, for example, the dynamics of different polymeric species.

Acknowledgment. Financial support by NATO Grant $0321 / 87$ and partial support by Bogazici University Research Fund, Project no. 89-P0041, are gratefully acknowledged. We thank Dr. L. Bokobza for stimulating discussions.

\section{References and Notes}

(1) Rouse, P. E. J. Chem. Phys. 1953, 21, 1272.

(2) Zimm, B. H. J. Chem. Phys. 1956, 24, 269.

(3) Bixon, M. J. Chem. Phys. 1973, 58, 1459.

(4) Zwanzig, R. J. Chem. Phys. 1974, 60, 2717.

(5) Doi, M.; Edwards, S. F. The Theory of Polymer Dynamics; Oxford University Press: New York, 1986.

(6) Jernigan, R. L. In Dielectric Properties of Polymers; Karasz, F. E., Ed.; Plenum: New York, 1972; p 99.

(7) Bahar, I.; Erman, B. Macromolecules 1987, 20, 1368.

(8) Bahar, I.; Erman, B.; Monnerie, L. Macromolecules 1989, 22, 2396.

(9) Kuhn, W.; Kuhn, H. Helv. Chim. Acta 1945, 28, 1533; 1946, 29, 7.
(10) Allegra, G. J. Chem. Phys. 1978, 68, 3600.

(11) Bahar, I.; Erman, B.; Monnerie, L. Macromolecules 1990, 23, 1174.

(12) Fixman, M. J. Chem. Phys. 1978, 69, 1527.

(13) Fixman, M. J. Chem. Phys. 1978, 69, 1538.

(14) Allegra, G.; Ganazzoli, F. J. Chem. Phys. 1981, 74, 1310.

(15) Allegra, G.; Ganazzoli, F. Macromolecules 1981, 14, 1110.

(16) Allegra, G.; Higgins, J. S.; Ganazzoli, F.; Lucchelli, E.; Brückner, S. Macromolecules 1984, 17, 1253.

(17) Ganazzoli, F.; Allegra, G.; Higgins, J. S.; Roots, J.; Brückner, S.; Lucchelli, E. Macromolecules 1985, 18, 435.

(18) Bahar, I. J. Chem. Phys. 1989, 91, 6525.

(19) Bahar, I.; Mattice, W. L. Macromolecules 1990, 23, 2719.

(20) Paul, E.; Mazo, R. M. Macromolecules 1971, 4, 424.

(21) Jernigan, R.; Szu, S. C. J. Polym. Sci., Polym. Symp. 1976, 54, 271.

(22) Kramers, H. A.; Wannier, G. H. Phys. Reu. 1941, 60, 252, 263.

(23) Flory, P. J. Statistical Mechanics of Chain Molecules; Interscience: New York, 1969. Reprinted by Hanser Publishers, Oxford University Press, 1989.

(24) The transition partition function $Z(\tau)$ present in the formulations of refs 18 and 19 is not explicitly included here since it is equal to 1, provided that single-bond time-delayed joint probabilities are defined according to eq 1 .

(25) Mashimo, S. Macromolecules 1976,9,91. Mashimo, S. J.Polym. Sci. Polym. Phys. Ed. 1981, 19, 213.

(26) Orwoll, R. A.; Stockmayer, W. H. Adv. Chem. Phys. 1969, 15, 305.

(27) Helfand, E. Science 1984, 226, 647.

(28) Weber, A.; Helfand, E. J. Chem. Phys. 1983, 87, 2881.

(29) Morawetz, H. Science 1979, 203, 405.

(30) Stockmayer, W. H.; Gobush, W.; Chikahisa, Y.; Carpenter, D. K. Faraday Discuss. 1970, 49, 182. 